\title{
A model for sensory adaptations of the visual system in squint
}

\author{
L. D. PICKWELL \\ From the Undergraduate School of Studies in Ophthalmic Optics, University of Bradford
}

SUMMARY Sensory adaptations to squint are considered in the light of present knowledge of the visual sensory system. A model to assist our understanding of the clinical presentation of sensory adaptation is proposed. Suppression and abnormal correspondence are considered as anomalies of different parts of the sensory system, and the interplay of these adaptations is reviewed.

In squint the embarrassment to binocular vision is considered to be of 2 types-diplopia and confusion. The object of regard has a central image in the fixing eye and a peripheral image falling on a noncorresponding area in the deviated eye. The object will therefore be located in 2 visual directions unless sensory adaptations occur. The location of the same object in 2 visual directions is diplopia. Confusion arises because in normal surroundings the fovea of the deviated eye will receive an image of some other object. If normal correspondence exists, the foveal image in the straight eye will be confused by this different foveal image from the deviated eye. Sensory adaptations occur to eliminate the diplopia and confusion. These adaptations are amblyopia, abnormal retinal correspondence, and suppression. The faulty pursuit of tracking movement that occurs in squint also may be due to the sensory adaptations.

Our understanding of the clinical presentation of these sensory adaptations has improved as new methods of investigation have been developed. ${ }^{1}$ The purpose of this paper is to further our general understanding of these conditions by presenting a simple model of the visual system from the evidence of more recent advances in electrophysiology. Such a model must necessarily be a great simplification, as a large proportion of the physiological evidence is not derived from human subjects but from a variety of different animals. Only broad generalisations can be made, and it would be inappropriate to be too specific about the details of the human visual system.

It has become clear that the nerve fibres from the retinal ganglion cells to the centres of the brain are

Correspondence to $\mathrm{Mr}$ L. D. Pickwell, Undergraduate School of Studies in Ophthalmic Optics, University of Bradford, Bradford, West Yorkshire BD7 1DP. of more than one type: those carrying the sustained response to a visual stimulus, the $x$ cell system, and those carrying a transient response, the $y$ cell system. ${ }^{2}$ The former seems to be associated with central form vision and acuity, while the y system seems to be associated with the peripheral retina and concerned with the location of objects in space which enables fixation movements to be made. The $x$ (sustained) system is concerned with what the object is, and the $y$ (transient) system with where it is. For the purpose of this model the $x$ system will therefore be referred to as the 'what system', and the more peripheral mechanism as the 'where system'.

There is also a deal of accummulated evidence for a third type of nerve cell associated with the central retina, the $w$ cell. The likely purpose of this seems to be in serving the pursuit eye movements, retaining a central image when the eye is following a moving object. ${ }^{3}$ In line with the other 2 systems, this $\mathrm{w}$ mechanism will be briefly called the 'pursuit system'.

Fig. 1 is a schematic representation of the 'what' and 'where' systems. Three receptor cells are shown in association with 3 ganglion cells. To avoid confusion of the diagram the retinal bipolar cells are not actually drawn. These may connect more than 1 receptor to a ganglion cell. This lateral conduction across the retina, by which receptor cells from a receptive area are associated with 1 ganglion cell, is mediated also by horizontal and amacrine cells. This will be called 'confluence' of nerve paths, and is represented in the diagram by 1 horizontal cell. For a particular receptor the laterally conducting cells may allow confluence on more than 1 ganglion cell, so that a single receptor could send signals to be used by the visual system in different ways. ${ }^{4}$ 
Fig. 1 Model of the visual sensory system showing the 'what' and 'where' systems.

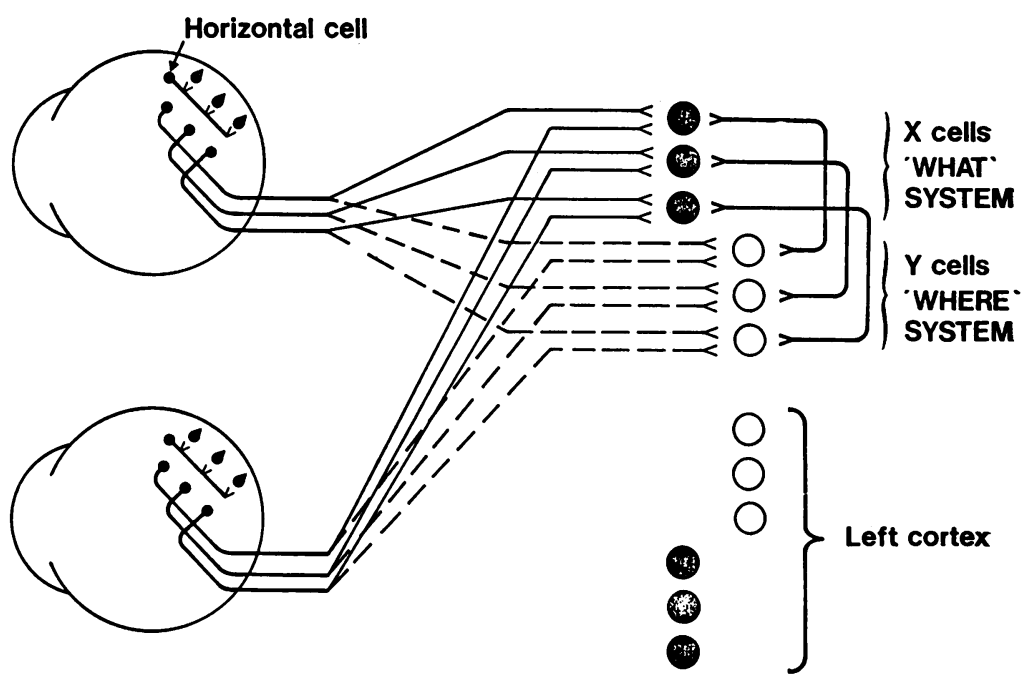

It is suggested that the nerve impulses from the ganglion cell layer are mediated by the visual pathways to 2 brain centres. ${ }^{5}$ (Schneider 1969). This is represented in the diagram by the division of the nerve fibres to the 2 groups of cells labelled 'what system' and 'where system'. The diagram is not intended to indicate the actual anatomical details of the human system, which is still speculative. In some animals there is evidence to suggest that the 'what system' is the visual cortex in the occipital lobes and the 'where system' is in the superior colliculus of the midbrain, ${ }^{67}$ the "where system' fibres leaving the visual pathway by the superior brachium just before the lateral geniculate body. In animals with forward-looking eyes and overlapping binocular vision, it appears likely that the visual cortex is also intimately concerned with the 'where system'. ${ }^{2}$ However, the model is not concerned with precise anatomical detail but with giving a working understanding of the visual system to help our understanding of sensory adaptation to squint.

There is evidence to suggest that association fibres connect the likely locations of the 'where' and the 'what' system, ${ }^{8}$ and these fibres are represented in the right upper part of the diagram. The cortical cells are shown as circles and receive fibres from both eyes, that is, they are binocularly driven cells. In the diagram they represent the simplest level of cortical cell. In order that form vision can be elaborated a number of these simple cells are connected to a single more complex cell, and a number of these to a hypercomplex cell ${ }^{10}$ (Fig. 2). This further confluence (not shown in Fig. 1) occurs in the 'what system', and it results in the receptive area for the hypercomplex cortical cells being very much larger than the retinal area connected to a single ganglion cell. In the process of form analysis, by the hypercomplex cells, the precise location of the image of the retina is lost, but localisation is not the concern of the 'what system'.

This is the simple model of the system. If we consider the sensory adaptations in terms of this dual system, we get a better insight into their nature

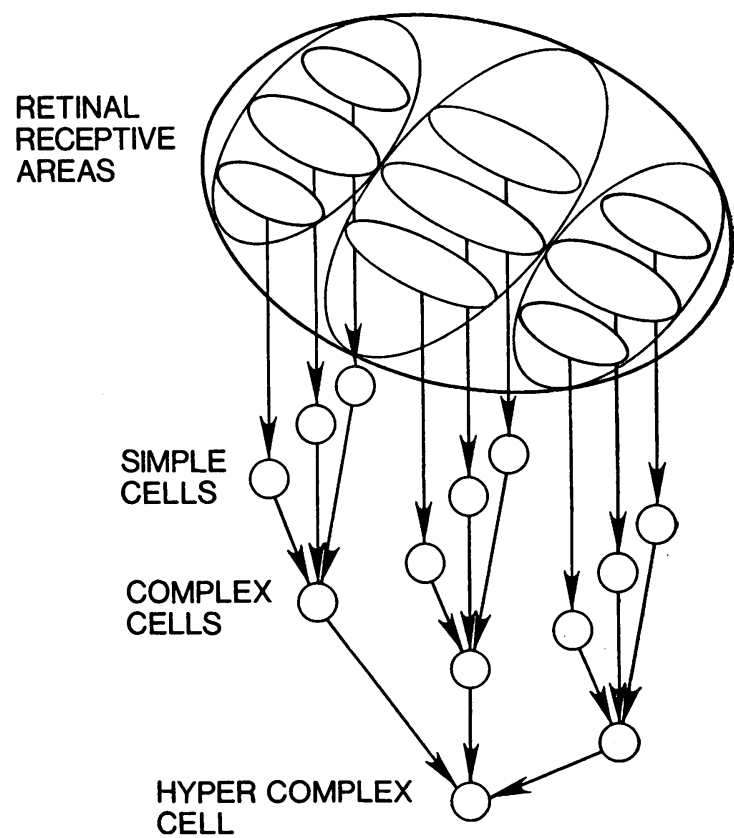

Fig. 2 Simple, complex, and hypercomplex cortical cells indicating the increase in retinal receptive areas with confluence of nerve paths. 




than if they are considered in terms of a single visual pathway as previously.

\section{Amblyopia}

It has already been suggested that amblyopia can be explained in terms of an increase in lateral conduction. ${ }^{11}$ A loss of acuity and form vision would occur if the 3 retinal receptors shown in Fig. 1 were to be connected to only 1 ganglion cell, or alternatively. if they were each connected to all 3 ganglion cells, which then sent the same signal to the associated cortex cells. This increased and abnormal confluence of nerve paths could occur in the retina and be mediated by the horizontal or amacrine cells as shown in Fig. 3, or it could occur at some higher point in the visual pathway. Although for simplicity of drawing the diagram the nerve fibres for both the 'what' and the 'where' systems are shown as coming from the same ganglion cells, they could, in fact, be separate ganglion cells for the 2 systems. If this were true, or if the confluence occurred at a higher point in the pathway, the confluence could affect the 'what system' and reduce acuity without the involvement of the "where system'. This would permit amblyopia with no other sensory adaptations, which we know occurs frequently.

\section{Suppression}

In addition to a change in the degree of confluence occurring in the system another modification can occur-that of inhibition of a signal at a nerve synapse. Confluence and inhibition are both im- portant aspects of normal sensory nerve processing, and their possible role in sensory adaptations has already been discussed. ${ }^{12}$ It is now suggested that abnormal inhibition can occur separately in that 'what' and the 'where' systems (Fig. 3). If there is inhibition of the 'what system' fibres from the squinting eye, this would result in suppression of form vision from that eye. This is represented in Fig. 3 by the black rectangles interposed at the synapse between the $x$ cell fibres from the squinting eye and the 'what system' cortical cells. The 'where system' could remain undisturbed. This would result in a squinting patient being unable to 'see' an object with the deviated eye, that is, no diplopia, but still being able to locate its direction.

This can be demonstrated clinically. If a patient with a large-angle squint (over $15^{\circ}$ ) is asked to look at a spot-light, this will be fixed with the dominant eye and the patient will report no second image. Where there is established suppression, no diplopia will be reported even if a red filter is placed before the straight eye and a green filter before the squinting eye. The patient will report that the green light cannot be seen. If the patient is then asked to turn the eyes to look at the green light, very many patients will be able to make a prompt and precise fixation movement to fix with the squinting eye. That is, the patient has turned the eyes to fix an object with the squinting eye even though this object could not be 'seen'. The 'what system' has been inhibited, but the 'where system' is still functioning. This seems to indicate the nature of suppression as an inhibition in the 'what system' which may leave the 'where system' intact. I believe that this is what occurs in squints with angles greater than 
about $12^{\circ}$. In such convergent deviations the retinal image of the squinting eye lies more peripherally than the disc area.

\section{Abnormal retinal correspondence}

In abnormal retinal correspondence the inhibition is in the nerve fibres from the ' $y$ ' ganglion cells of the squinting eye (Fig. 3). This would mean that the image can be detected with the 'what system' from both eyes, but the only information regarding its visual direction comes from the 'where system' of the undeviated eye. The image in the deviated eye is therefore given the visual direction determined by the 'where system' of the dominant eye. The 'what system' of the deviated eye is able to detect the form of the image (within the limits of its acuity), but owing to normal confluence in image analysing process the exact location of the image is unimportant within certain limits. These limits appear to be about $12^{\circ}$, as suppression is more likely than anomalous correspondence if the angle of squint is greater than this. It is also reasonable to assume that the very enlarged 'pseudo Panum's areas' first described by Bagolini in $1970,{ }^{1}$ can be explained by this area of inhibition or by abnormal confluence of nerve paths in the 'where system' from the squinting eye. In long-standing abnormal correspondence the dominance of the undeviated eye's 'where system' becomes more fixed, perhaps because long-standing inhibition eventually becomes intractable. Under these circumstances the dominance of the 'where system' of the straight eye is likely to remain even if the squint is straightened surgically or the deviation relieved by prisms.

Although these 3 adaptations may be considered separately, in most unilateral squint the total adaptation will consist of an interplay of all 3 . In very many cases, of alternating squint, however, the amblyopia may not be marked, and this suggests that inhibition in the 'where system' can occur independently of lateral conduction (or abnormal confluence) in that 'what system'.

\section{Pursuit anomalies}

It is a well known clinical observation that in many squints the ability of the eye to follow a moving target is impaired. This can be seen when undertaking the motility test for noncomitancy, not by any restriction of the total motor field, nor by an increase in the angle of squint in 1 direction, but by a failure of the deviating eye to follow the target. It may be assumed that this could be explained in terms of abnormal confluence or by inhibition of the 'pursuit system' ( $w$ cells). It is difficult to offer further explanation on this, as insufficient attention seems to have been given to analysing the anomalies of pursuit in squint.

\section{References}

${ }^{1}$ Bagolini B. Anomalous binocular vision and suppression in strabismic patients. Trans Int Ophth Optical Congress, Br Opt Asso, London, 1970; 298-308.

${ }^{2}$ Lindsay PH, Norman DA. Human Information Processing, 2nd ed. London: Academic Press, 1977; 237-8.

${ }^{3}$ Ikeda $\mathrm{H}$, Wright MJ. Is amblyopia due to inappropriate stimulation of the 'sustained' pathway during development? Br J Ophthalmol 1974; 58: 165-75.

${ }^{4}$ Michael CR. Retinal processing of visual images. $\mathrm{Sci}$ Am 1969; 220: 104-14.

${ }^{5}$ Schneider GE. Two visual systems. Science 1969; 163: 895-902.

${ }^{6}$ Robinson DA. Eye movements evoked by collicular stimulation in the alert monkey. Vision Res 1972; 12: 17951807.

${ }^{7}$ McIlwain JT. Visual receptive fields and their images in the superior colliculus of the cat, $J$ Neurophysiol $1975 ; 38$ : 219-30.

${ }^{8}$ Robinson DL, Goldberg ME. The visual substrate of eye movements: eye movements and the higher psychological functions. New Jersey: Erlbaun, 1978.

${ }^{9}$ Hubel DH, Weisel TN. Receptive fields and functional architecture in two non-striate areas of the cat. $J$ Neurophysiol 1965; 28: 229-89.

${ }^{10} \mathrm{Hubel}$ DN, Weisel TN. Anatomical demonstrations of columns in the monkey striate cortex. Nature 1969; 221: 749-50.

${ }^{11}$ Burian HM, von Noorden GK. Binocular Vision and Ocular Motility. St. Louis: Mosby, 1974; 245-6.

${ }^{12}$ Pickwell LD. How do squinters get rid of double vision? Optician 1977; 174: 21-3. 